\title{
Possible leakage radiation during malfunctioning of a Sagittaire accelerator
}

\author{
E. Woudstra*, H. Huizenga, J.A. van de Poel \\ Department of Clinical Physics, Dr Daniel den Hoed Cancer Center, Groene Hilledijk 301, 3075 EA Rotterdam, Netherlands
}

(Received 10 October 1992; revision received 25 June 1993; accepted 25 July 1993)

\begin{abstract}
A non-fatal accident with a CGR-Sagittaire accelerator in the Dr Daniel den Hoed Cancer Center (DDHCC) in Rotterdam in 1988 is described. In a period with frequently occurring technical problems, a patient, undergoing fractionated treatment at this accelerator for prostatic cancer, developed severe skin reactions on the right half ventral part of thorax, head and upper arm. The skin reactions suggested an irradiation with a dose of up to 10-20 Gy, so it was likely that a radiation accident had occurred caused by leakage radiation. A number of experiments were performed in attempting to explain the estimated large dose rates of leakage radiation. Under rather extreme malfunctioning conditions, a high leakage dose rate $(4 \mathrm{~Gy} / \mathrm{min})$ could be obtained during therapy use. This condition might have occurred during this period of technical difficulty.
\end{abstract}

Key words: Accelerator; Beam-transport; Leakage radiation; Malfunction; Radiation accident

\section{Introduction}

On 9 September 1988, a patient who had been treated in the radiotherapy department for prostatic cancer, was seen in the out-patient clinic a few days after he finished his treatment course. The patient had developed a severe skin reaction at the ventral part of the right arm, the right part of the thorax and the right part of the head (see Fig. 1). In addition he had lost some hair from his head. The skin reaction was investigated further, leading to the diagnosis of a radiation dermatitis caused by an estimated dose of 10-20 Gy. Apparently, a radiation accident had happened somewhere in the period of the fractionated treatment. The treatment had consisted of a course of $25 \times 2$ Gy via a 25-MV photon, 3-beam technique (anterior beam, combined with two lateral oblique beams at + and $-80^{\circ}$ ), produced by a CGRSagittaire, installed in 1974.

During the period this patient underwent radiotherapy treatment (August 1988), the Sagittaire showed a slow start at 'radiation on' and radiation was

\footnotetext{
* Corresponding author.
}

sometimes delivered at a dose rate that was less stable than normal, especially at some gantry angles, and sometimes the starting procedure failed completely. The temperature regulation in the modulator room was not functioning properly in this period. Qualified DDHCCand GE/CGR-technicians had immediately searched intensively for the origin of these problems, resulting in the replacement of temperature sensitive components of the beam monitor dosimetry-boards on 11 August. However, after the 11 August the instability problems remained and could only be solved by frequent readjustments of the pilot frequency.

During August 1988, the frequency of quality control checks was substantially increased. The functioning of the accelerator was monitored almost daily. Deviations from the normal beam properties (e.g., depth dose changes and significantly asymmetric beam profiles) were not observed. The interlock system had never been switched off during patient treatment. On 29 August 1988 the monitor chamber was replaced. The reason was a mechanical break-down whereby the chamber had been damaged and air-tightness had been lost. In addition, the temperature regulation in the modulator room 


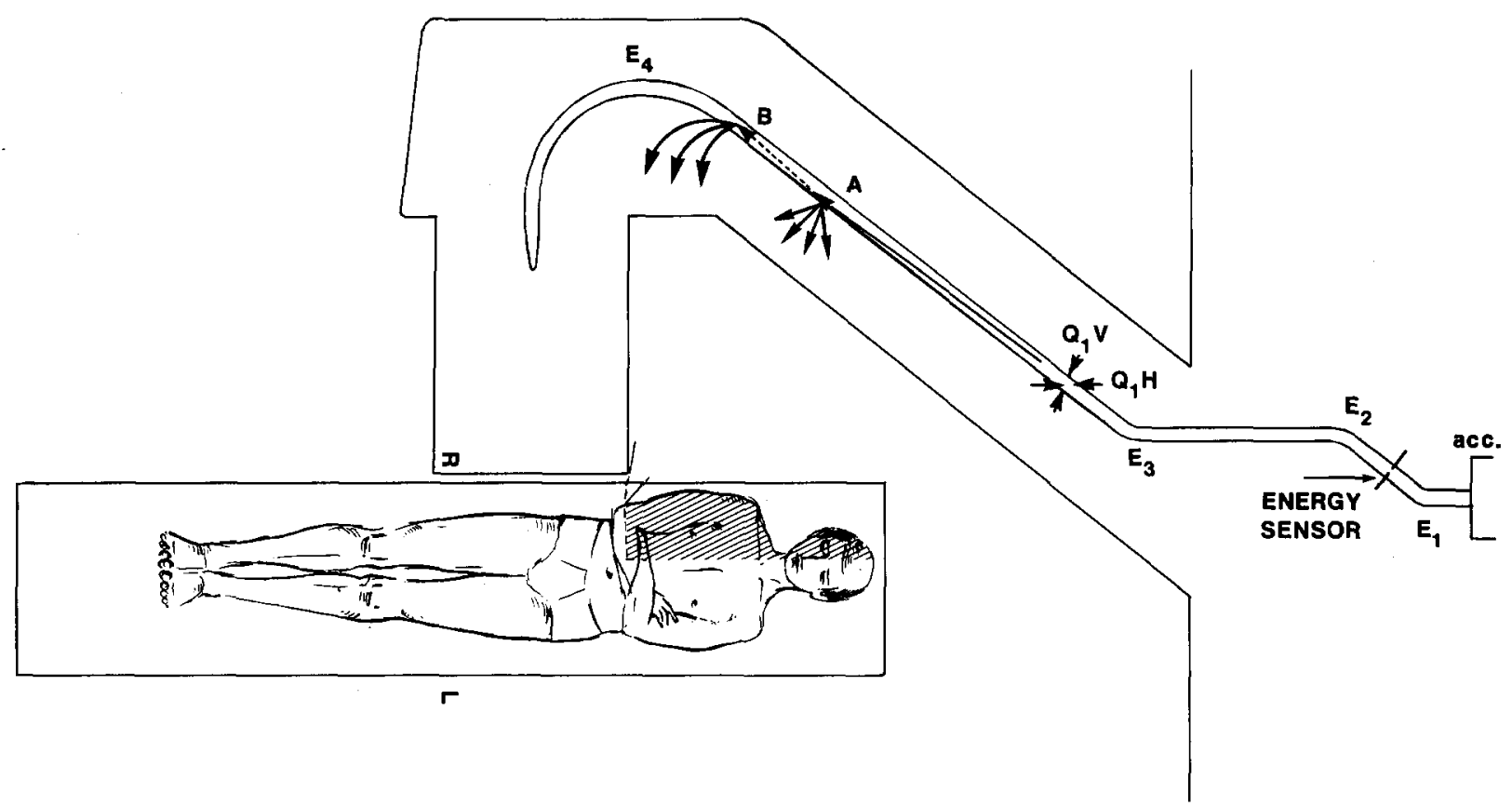

Fig. 1. Sketch indicating: patient position during irradiation with the most likely gantry position for the explanation of the skin reaction produced by leakage radiation (nearly horizontal at the right side of the patient); area of significant skin reaction observed after the irradiation period; a global drawing of the energy regulation and beam bending and transport system; and hypothesis for generation of leakage radiation.

was improved. From 30 August, the accelerator functioned again without problems.

Skin effects outside the radiation beam have not been observed on other patients in the described 'problem period'.

On 10 September 1988, after the diagnosis of a radiation dermatitis and the conclusion that a radiation accident had probably happened, the accelerator was taken out of clinical use for extensive investigations. It took 3 months to arrive at a plausible scenario for what had happened. This paper presents the results. The results have been reported to GE-CGR in January 1989. GECGR promised at that time, in writing, that they would inform all Sagittaire users immediately.

Detailed observations of the skin reaction of the patient, revealed that a skin reaction was not seen beyond the right ear and that the oral mucosis did not show any radiation reaction. Thus, the skin reaction could have been caused only by low energy radiation.

The most probable position and direction of the leakage radiation source was reconstructed from the region of skin reactions related to the most likely gantry and collimator position of the accelerator under the treatment set-up conditions. From this reconstruction it was concluded that the leakage radiation source was located at the upper end of the beam guiding tube close to the last part of the bending system (see Fig. 1). If the leakage radiation originated in the beam guiding tube it should have been able to penetrate the almost $3-\mathrm{cm}$ thick plastic gantry cover.
These observations have generated 3 questions:

- Is it possible to obtain a large dose rate of leakage radiation at the top of the beam guiding tube of the accelerator, which is not prevented by the interlock system?

- What are the misadjustments that cause such a malfunction?

- Could such conditions have happened occasionally in the period of instability during which the patient was treated?

\section{Materials and methods}

\subsection{The Sagittaire accelerator}

The Sagittaire consists of a microwave structure in which electrons can be accelerated to energies between 7 and $32 \mathrm{MeV}$. During this acceleration process, the electrons are kept together by focussing and centering coils. This basic accelerator structure is divided into two parts, the first of which yields electrons of about $7 \mathrm{MeV}$ and the second part is able to increase the energy up to $32 \mathrm{MeV}$.

This energy increase depends on the phase difference between the two parts. Energy regulation and beam transport is done in the system beyond the accelerator, where the electrons pass through an energy regulation part which consists of a bending magnet $\left(E_{1}\right)$ and an energy sensor which controls the input RF power (see Fig. 1.). 
Two additional bending magnets $\left(E_{2}\right.$ and $\left.E_{3}\right)$ lead the electrons into the beam guiding tube of the gantry and, finally, the bending magnet $\left(E_{4}\right)$ bends the beam into a strictly vertical direction through the head of the accelerator. The electron beam transport in the beam guiding part of the gantry is supported by centering magnets, $Q_{1} H$ and $Q_{1} V$, that lead the electrons to the input position for bending system $\mathrm{E}_{4}$.

The RF power is supplied by a klystron. The input wave for the klystron is generated by the master oscillator and synchronized by a $100-\mathrm{Hz}$ pulse generator. The beam monitor system consists of two ionisation transmission chambers, which are divided into two halves. The two parts of the two chambers are oriented at $90^{\circ}$ to one another in order to perform symmetry measurements in two beam directions. The current amplifiers of the centering magnets are controlled by means of the signals of these ionisation chamber parts. Papers on the characteristics of this accelerator have been presented by Briot and Dutreix [1] and Marinello et al. [3].

\subsection{Methods}

A significant amount of leakage radiation from the beam guiding tube is only possible if the beam has not been centered properly by the centering system in the beam guiding tube. Because the current amplifiers of the centering coils are controlled by the signals of the beam monitor system and the fact that 'imbalance' of the beam monitor system was observed and repair was carried out during the 'problem period', post-accident investigations were directed toward the function of the beam centering system related to the possible imbalanced sensitivity of the beam monitor system.

During the instability period of the accelerator, regular re-adjustments of pilot-frequency and offset voltages of the ionisation chamber circuits had been necessary to regain a temporary stability improvement. Therefore investigations have also been directed toward the influence of the RF-wave frequency on the beam centering system in the gantry.

A third possibility for electrons hitting the wall of the beam guiding tube is when the accelerated energy is not fully adapted to the bending magnet field strength used. Therefore, attention has been paid to the influence of changes to the bending system and the influence of changes to the energy of the accelerated electrons.

In more detail, the following list represents parameters that have been investigated for their possible influence on the generation of leakage radiation:

(a) beam centering system:

(i) centering magnets in accelerator tubes, $\mathrm{S}_{1} \mathrm{H}$, $\mathrm{S}_{1} \mathrm{~V}, \mathrm{~S}_{2} \mathrm{H}$ and $\mathrm{S}_{2} \mathrm{~V}$;

(ii) centering magnets in beam guiding tube, Q1H and Q1V, manually or by means of a balance change on the relevant dosimetry boards; (b) energy of accelerated electrons:

(i) energy-slit;

(ii) influence of RF-wave frequency and power;

(c) influence of bending system:

(i) partial short-circuit of bending magnets $E_{3}$ and $\mathrm{E}_{4}$.

Leakage radiation measurements have been performed with the accelerator functioning under non-normal conditions. An Alderson-Rando phantom was used to simulate a patient undergoing a prostate irradiation with a right lateral beam. Measurement positions have been selected in agreement with the position of the observed skin reactions of the mentioned patient at the surface of the phantom. The used gantry angles correspond with those that were used during the actual patient treatment. Regarding accelerator function two situations were defined:

- Strongly misadjusted parameters. The accelerator is not able to pass the starting procedure for 'beam on'. In this case, the measured ionisation represents the leakage dose generated during the starting phase.

- Moderate misadjustments. The accelerator generates a treatment beam with a low and unstable dose rate and almost maximum injector current. The measured ionisation rate should represent the leakage dose rate.

The radiation quality and geometric distribution of the leakage radiation at the phantom surface was investigated. Because the goal of this investigation was to estimate the likelihood of leakage radiation generation, no attempts have been made to measure the properties of the clinical beam (e.g. profile symmetry).

\subsection{Measurement equipment}

Dose and dose rate measurements for leakage radiation have been performed mainly by means of ionisation chambers. The positions of the chambers have been selected in agreement with the position of the observed skin reactions of the patient at the surface of the phantom. In order to ensure that all types of possible leakage radiation could be detected several types of ionisation chambers have been used. Mainly a NACP-chamber and a Baldwin Farmer (NE 2505/3B, nylon cap, graphite coating) without b.u.-cap and in some cases a PTWsurface chamber for low energy $X$-rays and a monitor for neutrons have been used.

\section{Theory}

The order of magnitude of a possible leakage dose rate can be estimated for the case of the full electron 
beam striking the wall of the beam guiding tube. This estimate contains two parts:

- an estimate of the electron beam intensity $I_{\mathrm{o}}$ [electrons/s] in the beam guiding tube, and

- an estimate of the scattered radiation fluence rate per unit of electron beam intensity $\Phi_{\mathrm{s}} / I_{\mathrm{o}}\left[\mathrm{cm}^{-2}\right]$.

The fluence rate at SSD $=100$ in a scanning $25-\mathrm{MeV}$ electron beam, under reference conditions (400 MU/ $\mathrm{min}, 1.6 \mathrm{cGy} / \mathrm{MU}$ at $D_{\max }$ ) can be easily calculated from the well-known relation between fluence and dose, and is approximately $3.3 \times 10^{8} \mathrm{~cm}^{-2} \mathrm{~s}^{-1}$, which, combined with the scanning area $\left(44 \times 44 \mathrm{~cm}^{2}\right)$ yields an electron beam intensity $I_{\mathrm{o}}$ of $6.4 \times 10^{11} / \mathrm{s}$. In a non-scanning 25 $\mathrm{MeV}$ electron beam the dose rate at $\mathrm{SSD}=100 \mathrm{~cm}$ under reference conditions is of course larger ( 400 $\mathrm{MU} / \mathrm{min}, 24 \mathrm{cGy} / \mathrm{MU}$ ), and the profile, which has been been measured with film at standard distance of $100 \mathrm{~cm}$ at the depth of maximum dose, has a Gaussian shape with a standard deviation of $2.8 \mathrm{~cm}$. This leads to an electron beam intensity $I_{\mathrm{o}}$ of $2.4 \times 10^{11} / \mathrm{s}$. This value is lower than for the scanning beam, since the scanning is performed over a larger area than the size of the monitor chamber. To generate a $25-\mathrm{MV}$ photon beam the electron beam intensity should be much larger than for a scanning electron beam since:

(a) the dose rate per fluence rate is larger for electrons than for photons (factor, 20),

(b) the efficiency of the target (factor, 2),

(c) the transmission of the flattening filter (factor, 5),

(d) the area over which the photon beam is spread, is a factor of 1.3 larger than the area of the scanning electron beam, and

(e) the dose per MU is a factor of 1.6 smaller in the photon beam.

This leads to an estimate for the electron beam intensity in the beam guiding tube of $I_{0}=1.0 \times 10^{14} \mathrm{e} / \mathrm{s}$.

Electron beam intensity measurements in an isolated lead block after switching off of the beam bending system with normal and maximal injector current yielded $0.6 \times 10^{14} \mathrm{e} / \mathrm{s}$ and $2.2 \times 10^{14} \mathrm{e} / \mathrm{s}$, respectively, which are of the same order of magnitude. A final estimate for the primary electron beam intensity is obtained from the combination of measurement and calculation:

$$
I_{\mathrm{o}}=1 \pm 0.5 \times 10^{14} \mathrm{e} / \mathrm{s} .
$$

The scattered radiation fluence rate per unit of electron beam intensity $\Phi_{\mathrm{s}} / I_{\mathrm{o}}\left[\mathrm{cm}^{-2}\right]$, for the case that the electron beam strikes the stainless-steel wall of the beam guiding tube, can be calculated as follows.

It is possible that the beam hits the beam guiding tube, and that the scattered radiation scatters directly to the patient. Suppose the electron beam within the beam guiding tube is incident on the wall $(1.5-\mathrm{mm}$ stainlesssteel) at a small angle ( $5^{\circ}$ or less). Due to scattering, the electrons will travel through $\sim 4 \mathrm{~mm}$ of steel. The angular spread of the beam will increase to $10-20^{\circ}$, the energy will decrease by $10 \mathrm{MeV}$, but the beam will emerge. In addition, secondary electrons are produced, with a much broader, almost diffusive character. The secondary electrons will penetrate the $3-\mathrm{cm}$ thick hard plastic gantry cover and will loose $5 \mathrm{MeV}$ of energy, and the angular spread will become even more diffuse. The number of secondary electrons per primary electron $I_{\mathrm{s}} / I_{\mathrm{o}}$ can be derived roughly under the assumption that all electrons with an energy of $13 \mathrm{MeV}$ or more will go straight on, and only the secondary electrons produced in the stainless steel with an energy between 5 and 13 $\mathrm{MeV}$ will penetrate the cover. According to Huizenga and Storchi [2], $I_{\mathrm{s}} / I_{\mathrm{o}}$ is, in this case, $0.2 \pm 0.1$. Under the assumption of full isotropic distribution of the secondary electrons, the fluence of the secondary electrons $\Phi_{\mathrm{s}} / I_{\mathrm{s}}$ at $100 \mathrm{~cm}$ follows, which combined with the value for $I_{\mathrm{s}} / I_{\mathrm{o}}$ yields:

$$
\Phi_{\mathrm{s}} / I_{\mathrm{o}}=1.7 \times 10^{-6} / \mathrm{cm}^{2} .
$$

This number can be considerably less if the assumption of isotropic scattering is not fullfilled.

Instead of this scenario, it can be supposed that $4 \%$ of the secondary electrons (estimated from the solid angle involved, 5-13 MeV) have reached the last bending magnet, which is not adapted to this lower energy. Therefore, an overly strong bending field directs these electrons to regions outside the clinical beam. If those electrons are spread over a $1-\mathrm{m}^{2}$ area in the patient plane, a contribution might arise of

$$
\Phi_{\mathrm{s}} / I_{\mathrm{o}}=0.8 \times 10^{-6} / \mathrm{cm}^{2} .
$$

The fluence/intensity ratio has been verified experimentally by directing a small electron beam $\left(1.8 \times 1.8 \mathrm{~cm}^{2}\right)$ to a $1.5-\mathrm{mm}$ thick stainless-steel plate, and measuring the scattered fluence rate at a distance of $100 \mathrm{~cm}$ behind a part of the gantry cover. The measurement results led to:

$$
\Phi_{\mathrm{s}} / I_{\mathrm{o}}=0.7-1.210^{-6} / \mathrm{cm}^{2} .
$$

A final estimate is obtained from the combination of measurement and calculation:

$$
\Phi_{\mathrm{s}} / I_{\mathrm{o}}=1 \pm 0.510^{-6} / \mathrm{cm}^{2} \text {. }
$$

Combination of Eqns. 1 and 2 yields a scattered fluence rate of approximately $10^{8} \mathrm{~cm}^{-2} / \mathrm{s}$, which in turn 
corresponds to a scattered dose rate of $3 \mathrm{cGy} / \mathrm{s}$, with a uncertainty of a factor of 3 .

This mainly theoretical consideration shows, that if the electron beam in the beam guiding tube strikes the wall, a scattered dose rate of $3 \mathrm{cGy} / \mathrm{s}$ of low energetic electrons might be delivered to a patient outside the treatment field. Such a situation, however, is expected to be prevented indirectly by the interlock system, because in this case the full electron intensity hits the wall of the beam guiding tube and it is expected that the monitor chamber will not measure dose and therefore the interlock system for dose absence will prevent this situation after about $8 \mathrm{~s}$. In case of a very low dose rate on the monitor chamber, a dose rate interlock system will prevent this at a chosen dose rate trip level.

\section{Results}

Leakage dose rates by accelerator misadjustments have been measured. None of the parameters mentioned in Methods (section 2.2., above) sufficiently influenced the production of leakage radiation to explain the observed skin reaction at the patient. Misadjustments mostly resulted in a simple stop of beam generation without an increased leakage radiation output. Only changes in the centering magnet currents $\left(\mathrm{Q}_{1} \mathrm{H}\right.$ and $\left.\mathrm{Q}_{1} \mathrm{~V}\right)$ showed a significant increase in leakage dose rate. However, the combination of a deviated current through the centering magnet $Q_{1} V$ with a changed pilot oscillator frequency, forced the accelerator to produce a large dose rate of leakage radiation (about $4 \mathrm{~Gy} / \mathrm{min}$ ) at a very low output rate of only $20 \mathrm{MU} / \mathrm{min}$ and a very large injector current $(250 \mu \mathrm{A})$. This dose rate has been measured at the surface of the Rando phantom simulating the position of the patient. In this case the changed $Q_{1} V$ current was generated using the automatic centering system and a changed balance adjustment of the dosimetry boards. Despite this malfunction the accelerator was even able to reach 'beam on' without the activation of the interlock circuits and showed a quite stable behaviour. The geometric leakage radiation pattern was quite comparable with the radiation pattern observed on the patient skin. A sheet of 5$\mathrm{mm}$ and 25-mm PMMA reduced the dose rate to 50\% and $10 \%$, respectively, of the incident dose rate, which demonstrates a low average radiation energy.

Some additional remarks must still be made.

Although this investigation has demonstrated that the Sagittaire might generate a quite large amount of leakage radiation, without activation of safety interlocks, these results do not exclude other possible causes for the described accident. It should be emphasized that it has not been possible to completely reconstruct the accident and to derive the estimated dose value as obtained from the skin observations from the measured leakage dose rate values. It is, for example, not clear whether the patient received the skin dose during one or several fractions. However, the coincidence of the required misadjustments for the generation of a large leakage dose rate can be argued to have happened occasionally in the 'problem period' during which the patient has been irradiated. In this period, cooling problems regarding the modulator room might have caused frequency changes. The $Q_{1} V$ misadjustment could have been due to the observed imbalance in the dosimetry system that was repaired during the 'problem period'. This discussion supports the idea that these misadjustments might have happened occasionally in that period and might have been the reason for the skin dose on that particular patient.

It became clear that this patient had mentioned an extreme skin sensitivity for (for example) sunlight, and in view of this fact the estimated skin dose should probably be lower.

The large amount of leakage radiation was generated under a very low dose rate, measured by the monitor system. This condition for a large amount of leakage radiation could be obtained only by too low a dose rate interlock level (20 MU/min) and had not happened in the case of an interlock level of, for example, 50 $\mathrm{MU} / \mathrm{min}$. This emphasizes again the common knowledge that properly adjusted dose rate interlock systems will prevent an accelerator from functioning under $a b-$ normal and dangerous conditions.

Apart from the use of a dose rate interlock circuit, an additional precaution might be the use of a lead shield along the beam guiding tube on the side of the patient. In this case it might have largely decreased the dose rate of this leakage radiation to the patient. Moreover, an additional leakage radiation measuring system close to the unshielded beam guiding tube might also have prevented this accident.

Because the accelerator had already been in use for 15 years, the Sagittaire was taken out of clinical use and has been replaced by another type of accelerator.

In may 1989 the CGR-GE-company has assured the Daniel den Hoed Cancer Center that it would inform other Sagittaire users in writing about this accident, diagnosis and our opinion regarding necessary precautions to be taken.

\section{Conclusions}

It has been demonstrated that despite the relatively high degree of control and the security systems, this old Sagittaire (1974) could be forced into an error condition due to a dose rate interlock level that was too low, and hence could have produced a large amount of leakage radiation.

The measurements and estimations regarding the ac- 
tual electron current as well as regarding leakage dose rate agree in order of magnitude.

The observed skin reactions of the patient can only be related to the maximum measured leakage dose rate if an extremely high skin sensitivity is assumed.

\section{References}

1 Briot, E. and Dutreix, A. Dosimétrie de faisceaux d'électrons de haute énergie d'un accéléateur linéaire, J. Radiol. Electrol. 57(5): 447-454, 1976.

2 Huizenga, H. and Storchi, P.R.M. Numerical calculation of energy deposition by broad high energy electron beams, Phys. Med. Biol. 34: 1371-1396, 1989.

3 Marinello, G., Dutreix, A. and Chapuis, G. Etude, de l'effacité de collection des chambres d'ionisation cylindriques irradiées dans les faisceaux de rayonnement pulsé d'un accélérateur linéaire, $\mathbf{J}$. Radiol. Eletrol. 57(11): 789-800, 1976. 